\title{
TRIGONOMETRIC PARALLAXES OF PLANETARY NEBULAE
}

\author{
J. R. PIER, H. C. HARRIS, C. C. DAHN and D. G. MONET \\ U.S. Naval Observatory Flagstaff Station, U.S.A.
}

\begin{abstract}
Parallaxes are presented for nine Planetary Nebulae central stars.
Nine planetary nebulae fields are now routinely observed on the U.S. Naval Observatory CCD trigonometric parallax program (Monet et al, 1992). Due to the small parallaxes of the PN central stars, the corrections from relative to absolute parallax are crucial. To aid in this correction, spectroscopic and/or photometric parallaxes were obtained for all of the reference frame stars. Reasonable solutions are beginning to emerge for three of the central stars, while the remaining fields will require many more frames before a parallax solution can be given with any comfortable degree of certainty.

Results are presented in the Table below. The PN field identification is given in the first column, column 2 lists the number of CCD frames used in the solution, the third column gives the epoch range covered by the frames employed and the fourth column lists the absolute parallax and mean error in the parallax (in milliarcseconds) for the central star. The absolute $\mathrm{V}$ magnitude in the fifth column is based upon the absolute parallax and CCD photometry. The comment appearing in the last column is a qualitative assessment of the stability of the solution based upon the repeatability of the results as various parameters (removing certain frames and/or reference stars) were changed from solution to solution.
\end{abstract}

Trigonometric Parallaxes of Planetary Nebulae Central Stars

\begin{tabular}{|c|c|c|c|c|c|}
\hline PN & \#Frames & Epoch & $\Pi \pm$ m.e. (mas) & $\mathrm{M}_{V}$ & Comment \\
\hline \hline A 21 & 35 & $89.2-92.1$ & $2.9 \pm 0.6$ & 8.3 & Fragile \\
\hline A 24 & 12 & $88.0-92.1$ & $4.0 \pm 1.6$ & - & Insufficient Data \\
\hline A 29 & 18 & $89.2-92.3$ & $-1.0 \pm 2.2$ & - & Insufficient Data \\
\hline A 31 & 18 & $88.1-92.3$ & $4.9 \pm 1.7$ & 9.0 & Preliminary \\
\hline A 74 & 48 & $88.5-92.5$ & $1.2 \pm 0.8$ & $7 .:$ & Preliminary \\
\hline NGC 6720 & 142 & $88.4-92.5$ & $2.0 \pm 0.6$ & 7.3 & Fragile \\
\hline NGC 6853 & 116 & $88.5-92.5$ & $2.8 \pm 0.5$ & 6.3 & Stable \\
\hline PW 1 & 43 & $87.9-92.1$ & $3.1 \pm 0.7$ & 8.0 & Stable \\
\hline S 216 & 57 & $89.7-92.1$ & $8.3 \pm 1.4$ & 7.3 & Stable \\
\hline
\end{tabular}

\section{Reference}

Monet, D.G., Dahn, C.C., Vrba, F.J., Harris, H.C., Pier, J.R., Luginbuhl, C.B. \& Ables, H.D.: 1992, Astron.J. 103, 638 . 